\title{
Desferal regulates hCtr1 and transferrin receptor expression through Sp1 and exhibits synergistic cytotoxicity with platinum drugs in oxaliplatin-resistant human cervical cancer cells in vitro and in vivo
}

\author{
Szu-Jung Chen ${ }^{1,2}$, Ching-Chuan Kuo ${ }^{3}$, Hsin-Yi Pan ${ }^{2}$, Tsui-Chun Tsou4 ${ }^{4}$ Szu-Ching \\ Yeh $^{4}$, Jang-Yang Chang ${ }^{1,2,5}$ \\ ${ }^{1}$ Institute of Clinical Pharmacy and Pharmaceutical Sciences, College of Medicine, National Cheng Kung University, Tainan, \\ Taiwan, ROC \\ ${ }^{2}$ National Institute of Cancer Research, National Health Research Institutes, Tainan, Taiwan, ROC \\ ${ }^{3}$ Institute of Biotechnology and Pharmaceutical Research, National Health Research Institutes, Zhunan, Taiwan, ROC \\ ${ }^{4}$ Division of Environmental Health and Occupational Medicine, National Health Research Institutes, Zhunan, Taiwan, ROC \\ ${ }^{5}$ Division of Hematology/Oncology, Department of Internal Medicine, National Cheng Kung University Hospital, College of \\ Medicine, National Cheng Kung University, Tainan, Taiwan, ROC
}

Correspondence to: Jang-Yang Chang, email: jychang@nhri.org.tw, z10208083@email.ncku.edu.tw

Keywords: oxaliplatin resistance, copper transporter hCtr1, transferrin transporter TfR1, desferal, specificity protein 1 Sp1

Received: April 11, 2016

Accepted: June 13, 2016

Published: June 30, 2016

\section{ABSTRACT}

The development of resistance to platinum drugs in cancer cells severely reduces the efficacy of these drugs. Thus, the discovery of novel drugs or combined strategies to overcome drug resistance is imperative. In addition to our previous finding that combined D-penicillamine with platinum drugs exerts synergistic cytotoxicity, we recently identified a novel therapeutic strategy by combining an iron chelating agent desferal with platinum drugs to overcome platinum resistance in an oxaliplatin-resistant human cervical cancer cell line, S3. Further study demonstrated that the level of platinum-DNA adduct formation positively correlated with cell death in combination of desferal with platinums than that of each drug alone in S3 cells. Decrement of human copper transporter 1 (hCtr1) and transferrin receptor 1 (TfR1) expression involved in the development of platinum resistance in $\mathbf{S} 3$ cells. Moreover, desferal promoted the expression of hCtr1 through the upregulation of Sp1. The overexpression of Sp1 increased the expression of NF-KB and translocated it into the nucleus to bind to the TfR1 promoter region, which subsequently increased the expression of TfR1. Importantly, the cotreatment of oxaliplatin with desferal significantly potentiated the oxaliplatin-elicited antitumoral effect in the oxaliplatin-resistant xenograft animal model without any toxic effect observed. Taken together, these results demonstrated that the combination of desferal with oxaliplatin can overcome oxaliplatin resistance through the regulation of hCtr1 and TfR1, and may have beneficial effect for treatment of patient with oxaliplatin-refractory tumors.

\section{INTRODUCTION}

The platinum-based drugs, included cisplatin, oxaliplatin, and carboplatin, were the most commonly used therapeutic agents for treating solid tumors in clinical [1]. However, accumulating evidences indicated that the development of drug resistance severely impedes and reduces the antitumor effect of these agents $[2,3]$.
Platinum-based drugs cause cell death through the formation of a covalent bond between the platinum moiety of a platinum-based drug and the purine base of DNA [4]. These intra- and inter-strand crosslinks of platinum-DNA complexes destroy the DNA structure, resulting in cell death [5]. Studies have reported that reduction in the intracellular accumulation of platinum-DNA adduct through the regulation of drug influx and efflux pumps, 
enhancement of DNA repair and drug detoxification, are major factors contributing to platinum resistance $[5,6]$.

Platinum drugs enter cells through diffusion or endocytosis. Studies have reported that human copper transporter 1 (hCtr1), a copper influx transporter, participates in the transportation of platinum drugs and is frequently downregulated in platinum-drug resistant variants $[7,8]$. Two copper efflux transporters, coppertransporting ATPase 1 (ATP7A) and copper-transporting ATPase 2 (ATP7B), transport platinum drugs from the cytoplasm into subcellular compartments, primarily localized to the trans-Golgi network for subsequent efflux in a similar manner to their effect on copper [9]. This action reduces the cytotoxicity of platinum drugs and causes drug resistance. We and others have demonstrated that the decreased expression of $\mathrm{hCtr} 1$ and overexpression of ATP7A and ATP7B have a crucial role in the development of platinum resistance [10-14]. Moreover, our previous study reported that combination of D-penicillamine, a copper chelator, and platinum drugs exhibits synergistic interaction in oxaliplatin-resistant cancer cells through modulation of hCtr1 and ATP7A expression $[13,14]$. Thus, we proposed that the metal chelators, such as iron chelators, may also be benefit in manipulation of copper transporters and sensitize resistant cancer cells with chemotherapeutic agents. In the present study, we first time demonstrated that combination of desferal, an iron-chelating agent, with platinum drugs synergistically inhibits tumor growth and promotes tumor cell death in oxaliplatin-resistant cells in vitro and in preclinical human xenograft animal model. The underlying mechanism for this synergism, at least in part, is through the upregulation of hCtr1 and transferrin receptor 1 (TfR1) by desferal, thereby subsequently increases the platinumselicited cellular platinum-DNA adduct formation and cytotoxicity.

\section{RESULTS}

\section{Combination effect of platinum drugs and desferal on SiHa and $\mathrm{S} 3$ cells}

Oxaliplatin-resistant cells S3 were established from $\mathrm{SiHa}$ cells by exposure to increasing concentrations of oxaliplatin. The antiproliferative effect of platinum-based drugs and desferal in SiHa and S3 cells was illustrated in Table 1. As compared with parental SiHa cells, S3 cells were more resistant to oxaliplatin, followed by cisplatin and carboplatin, with the resistance index of approximately $77.6,12.7$, and 2.7 , respectively. However, the $\mathrm{IC}_{50}$ value of desferal for both $\mathrm{SiHa}$ and $\mathrm{S} 3$ cells was almost similar.

To investigate the combination effect of desferal and platinum-based drugs, both $\mathrm{SiHa}$ and $\mathrm{S} 3$ cells were simultaneously treated with desferal and platinum-based drugs for $72 \mathrm{~h}$. As listed in Table 2, the combination index
(CI) of desferal with oxaplatin, cisplatin, and carboplatin was $0.51,0.68$, and 0.93 in S3 cells and 1.76, 1.18, and 1.37 in parental $\mathrm{SiHa}$ cells, respectively. We further determined whether the increased sensitivity of S3 cells toward platinums correlated to the increased the formation of intracellular platinum-DNA adduct. As the result, the formation of platinum-DNA adduct significantly increased in the combination regimens with desferal than platinum alone in S3 cells; however, this effect did not observe in parental cells (Figure 1A-1C). Compared with a singledrug treatment, the cotreatment of desferal with oxaliplatin or cisplatin significantly increased DNA adducts formation in $\mathrm{S} 3$ cells. However, no change in DNA adduct formation was observed in $\mathrm{S} 3$ cells treated either with carboplatin plus desferal or carboplatin alone (Figure 1D).

\section{Desferal increases the expression of hCtr1 and TfR1 through the upregulation of Sp1 in S3 cells}

To determine the basal level of both hCtr1 and TfR 1 proteins in SiHa and $\mathrm{S} 3$ cells, western blot analysis was performed. The results revealed that the expression level of hCtr1 and TfR1 was higher in SiHa cells than in S3 cells (Figure 2A). Furthermore, we examined the effect of desferal on intracellular iron and copper concentrations in both $\mathrm{SiHa}$ and $\mathrm{S} 3$ cells. As presented in Figure 2B, desferal treatment significantly reduced more intracellular iron and copper concentrations in S3 cells than in SiHa cells $(48 \%$ vs. $17 \%, P=0.019 ; 96 \%$ vs. $69 \%, P=$ 0.012). Previously, we demonstrated that the expression of hCtr1 is controlled by the transcription factor Sp1, which is negatively regulated by intracellular copper concentrations [14]. We further evaluated the effect of desferal on the expression of Sp1 and hCtr1 in both SiHa and $\mathrm{S} 3$ cells. We observed an increase in the expression of $\mathrm{Sp} 1$ and hCtr1 in the concentration- and time-dependent manner in S3 cells; however, desferal did not affect Sp1 and hCtr1 in SiHa cells (Figure 2C). Notably, desferal increased the expression of TfR1 in a time-dependent manner (Figure 2C). We further investigated whether the expression of hCtr1 is through the transcriptional regulation of Sp1 on the hCtr1 promoter in S3 cells. We observed that desferal treatment induced the binding of $\mathrm{Sp} 1$ to the hCtr1 promoter and increased the expression of hCtr1 (Figure 2D).

\section{Desferal treatment induces the expression of TfR1 through the Sp1-NF-кB p65-dependent pathway}

Several studies have reported that $\mathrm{Sp} 1$ regulates NF- $\mathrm{KB}$ p65 transcription $[15,16]$. As presented in Figure 3A, our results revealed that desferal increased the expression of NF- $\mathrm{kB}$ p 65 in S3 cells. Furthermore, we observed an increase in the nuclear expression of Sp1 and NF- $\mathrm{KB}$ p65 after treating $\mathrm{S} 3$ cells with desferal (Figure 3B). The nuclear accumulation 
Table 1: Sensitivity of parental SiHa cells and oxaliplatin-resistant S3 subline cells to platinumbased drugs and desferal

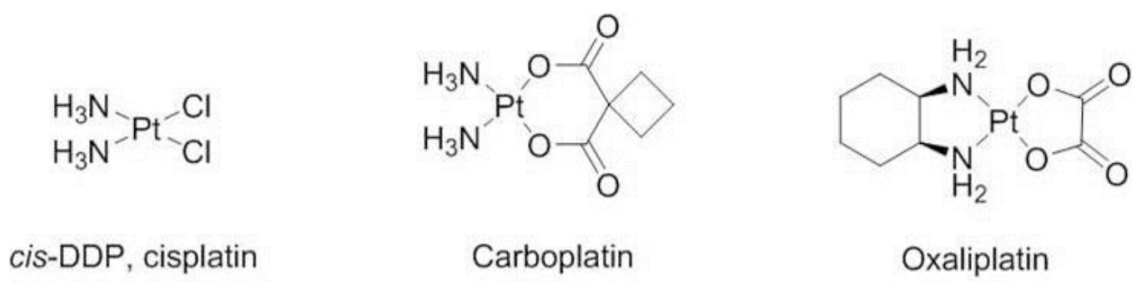<smiles></smiles>

\begin{tabular}{|c|c|c|c|}
\hline \multirow{2}{*}{ Drugs } & $\mathrm{SiHa}$ & S3 & \multirow{2}{*}{$\begin{array}{c}\text { Resistance Index } \\
\left(\mathrm{IC}_{50} \text { of } \mathrm{S} 3 / \mathrm{IC}_{50} \text { of } \mathrm{SiHa}\right)\end{array}$} \\
\hline & $\mathrm{IC}_{50}(\boldsymbol{\mu M})$ & $\mathrm{IC}_{50}(\boldsymbol{\mu M})$ & \\
\hline Oxaliplatin & $0.8 \pm 0.1$ & $62.1 \pm 1.6$ & 77.6 \\
\hline Cisplatin & $1.2 \pm 0.1$ & $15.2 \pm 1.5$ & 12.7 \\
\hline Carboplatin & $3.0 \pm 0.8$ & $8.1 \pm 0.6$ & 2.7 \\
\hline Desferal & $74.7 \pm 0.4$ & $77.0 \pm 1.6$ & - \\
\hline
\end{tabular}

Each value is presented as the means \pm standard deviation (S.D.) of three independent experiments.

Table 2: Combination effect of desferal and platinum drugs, including oxaliplatin, cisplatin, and carboplatin, on $\mathrm{SiHa}$ and $\mathrm{S} 3$ cells

\begin{tabular}{|c|c|c|}
\hline \multirow{2}{*}{\multicolumn{1}{c|}{ Drugs }} & \multicolumn{2}{c}{ SiHa } \\
\cline { 2 - 3 } \multicolumn{1}{c|}{ Combination Index (CI) } \\
\hline Oxaliplatin + Desferal & 1.76 & 0.51 \\
\hline Cisplatin + Desferal & 1.18 & 0.68 \\
\hline Carboplatin + Desferal & 1.37 & 0.93 \\
\hline
\end{tabular}

The drug combination analysis was performed using the Chou and Talalay method. Graphs representing values of combination index (CI) versus fractional effect were automatically generated using CalcuSyn software. Effect is the fraction of cell death induced by drug treatment and ranges from 0 to 1 , with 0 representing no cell death and 1 representing $100 \%$ cell death. The quantitative $\mathrm{CI}$ value defines addictive effect as $\mathrm{CI}=1$, synergism as $\mathrm{CI}<1$, and antagonism as $\mathrm{CI}>1$ in a combination of two drugs.

of NF-kB p65 maybe related to its transcriptional activity after desferal treatment. Moreover, the activation of NF- $\mathrm{kB}$ p65 in LPS-IFN $\gamma$-treated macrophage cells was reported to be relative to the expression of TfR1 [17]. Therefore, we analyzed the TfR1 promoter sequence by using the QIAGEN EpiTect chromatin immunoprecipitation (ChIP) promoter binding site prediction and observed the presence of potential NF- $\mathrm{kB}$-binding sites in the TfR1 promoter region. We hypothesized that the upregulation of TfR 1 is through

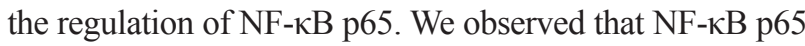
promoter-bound $\mathrm{Sp} 1$ and TfR1 promoter-bound NF- $\mathrm{KB}$ p65 were increased in S3 cells treated with desferal (Figure 3C). However, this effect did not observe in $\mathrm{SiHa}$ cells. In addition, the transcription levels of TfR1 and NF- $\mathrm{kB}$ p65 significantly increased after desferal treatment in S3 cells (Figure $3 \mathrm{C}$ ). To further determine whether $\mathrm{Sp} 1$ is responsible for the regulation of NF- $\mathrm{KB}$ p 65 and TfR1 expression, we knocked down Sp1 expression by using a siRNA followed by treated cell with desferal. As the result, we found that the silence of $\mathrm{Sp} 1$ reduced the expression of $\mathrm{TfR} 1$ in S3 cells in both presence and absence of desferal conditions (Figure 3D). 


\section{TfR1 serves as a transporter for platinum drugs}

To investigate whether TfR1 is responsible for the transportation of platinum-based drugs, we knocked down the expression of TfR1 by using a siRNA in both SiHa and S3 cells, followed by treatment with platinum-based drugs, and subsequently measured platinum-DNA adduct formation. As presented in Figure 4A, platinum-DNA adduct formation significantly decreased in both $\mathrm{SiHa}$ and $\mathrm{S} 3$ cells after the downregulation of TfR1. We further compared DNA adduct formation after treatment with platinum-based drugs in hCtr1 or TfR1 knockdown cells. We observed that the downregulation of hCtr1 and TfR1 reduced platinum-DNA adduct formation (Figure 4B). Among them, silencing of TfR1 is more effective than knockdown of hCtr1 to reduce platinum-DNA adduct formation.

\section{Desferal increases the antitumor effect of oxaliplatin in an oxaliplatin-resistant tumor xenograft model}

Our results support the notion that desferal increases the uptake of platinum-based drugs, which subsequently increases DNA adduct formation and sensitizes cells to death in vitro. We further examined the combination effect of desferal and oxaliplatin in vivo. Both $\mathrm{SiHa}$ and $\mathrm{S} 3$ tumor xenograft mouse models were used. Compared with vehicle controls, oxaliplatin delayed growth of both $\mathrm{SiHa}$ and S3 tumors. However, the antitumor effect increased
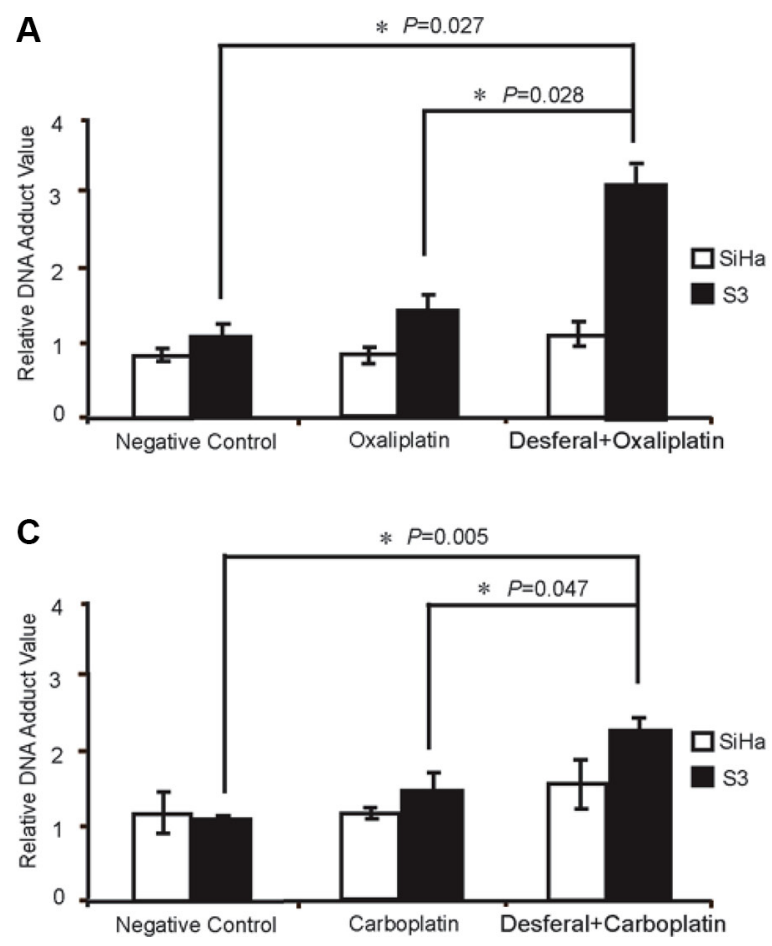

only in $\mathrm{S} 3$ cells after the combination treatment of desferal with oxaliplatin (Figure 5B). In addition, we observed no significant body weight loss in these mice (Figure 5A-5B). We further assessed the expression levels of hCtrl and TfR1 in tumor tissues through immunohistochemistry (IHC) staining. Consistent with our in vitro findings, desferal treatment considerably increased the expression of hCtr1 and TfR1 in S3 tumors (Figure 5C-5D). Taken together, our results demonstrate that the combination of desferal and oxaliplatin is an effective approach to inhibit the tumor growth in oxaliplatin-refractory tumors.

\section{DISCUSSION}

In our previous study, we demonstrated that a copper-chelating agent could reverse oxaliplatin resistance through the regulation of the copper importer hCtr1 and exporter ATP7A [14]. We further observed that the expression level of TfR1 was lower in S3 cells than in parental cells (Figure 2A). These findings prompted us to identify iron-chelating agent and investigate the combination effect of iron chelating agents with platinum drugs in oxalipaltin resistant tumor models. Indeed, we noted that desferal, an iron-chelating agent, exhibited synergistic interaction with oxaliplatin or cisplatin to inhibit tumor cell growth in oxaliplatin-refractory tumors.

Iron is an essential element for cell replication, metabolism, and growth. When iron is insufficient, cells increase TfR1 expression to import more iron into the
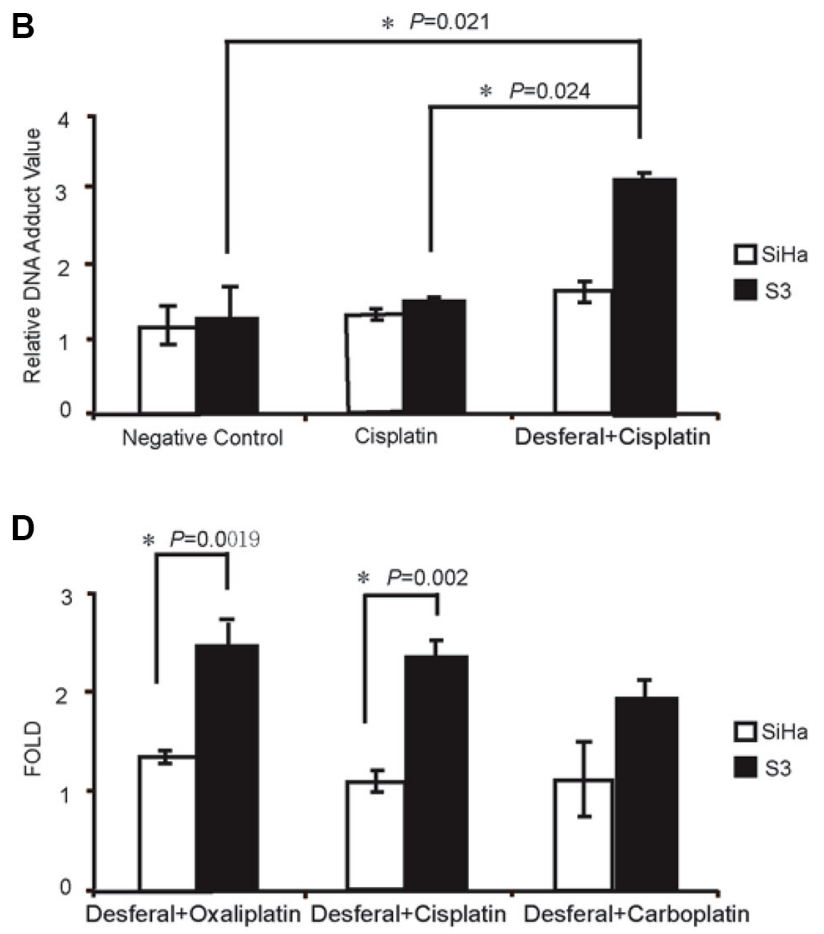

Figure 1: Desferal synergistically interacts with platinum drugs and increases platinum-DNA adduct formation in oxaliplatin-resistant $\mathbf{S 3}$ cells. (A-D) Measurement of platinum-DNA adduct formation in cells treated with 1-fold of $\mathrm{IC}_{50}$ of platinum drugs (refer to Table 1) and desferal, as described in the Materials and Methods section. Each value is presented as the mean \pm standard deviation (SD) of three independent experiments $(P<0.05)$. 
cell. Desferal, an iron chelator, is currently used to treat iron-overload diseases $[18,19]$. The potential of desferal and its analogs in cancer therapy has emerged from the finding that cancer cells require higher iron concentrations during DNA synthesis and growth than normal cells do [20]. Studies have reported that desferal removes iron from cancer cells and prevents iron uptake by TfR1, thus exerting the antiproliferative effect $[21,22]$. In addition to its iron-chelating effect, desferal can eliminate free copper. Van Reyke et al. reported that under optimal conditions, one mole of desferal is sufficient to reduce three moles of copper [23]. In an animal study, another iron chelating drug, deferasirox, increased copper levels by 2-fold in the kidney compared with those in the tumor. The copperdeferasirox complex was trapped in the kidney during the filtration, resulting in a significant increase in copper levels in the kidney $[21,22]$. These findings suggest that these compounds exhibit multi-chelating activity, which broadens their antitumor effect alone or in combination with other anticancer agents. Consistent with these findings, our data further confirmed that desferal has multi-chelating activity (Figure 2B), which synergizes with the effect of platinum-based drugs to treat cancer.

Platinum drugs mainly enter cells through passive diffusion or endocytosis. The net intracellular level of platinum drugs is balanced between influx and efflux. Studies have reported that several transporters have different functions to regulate the net intracellular level of platinum drugs [24, 25]. Evidence has indicated that copper transporters have a crucial role in transporting platinum drugs into cells $[8,26]$. The major copper influx transporter hCtr1 has been reported to assist the uptake of CDDP, carboplatin, and oxaliplatin and to regulate their cytotoxicity in yeast and mammalian cells $[27,28]$. Furthermore, two copper efflux transporters, ATP7A and ATP7B, transport platinum drugs from the cytoplasm into subcellular compartments, primarily localized to the transGolgi network for subsequent efflux in a similar manner to their effect on copper [9]. This action reduces the cytotoxicity of platinum drugs and causes drug resistance. We have previously demonstrated that the downregulation of hCtr1 and upregulation of ATP7A render cells resistant to platinum drugs [29]. In addition to the alteration of copper transporters, we observed that the expression level of TfR1 was lower in S3 cells than in parental cells (Figure 2A). These results prompted us to investigate whether TfR 1 has a role in transporting platinum drugs. The downregulation of TfR1 by using a siRNA reduced the formation of intracellular platinum-DNA adduct (Figure 4A). Moreover, the reduction in DNA adduct formation was significantly higher in cells treated with siTfR1 than in cells treated with sihCtr1. This is the first study to report that TfR1 can transport platinum drugs into cells, probably with a higher efficiency than that of hCtr1.

A
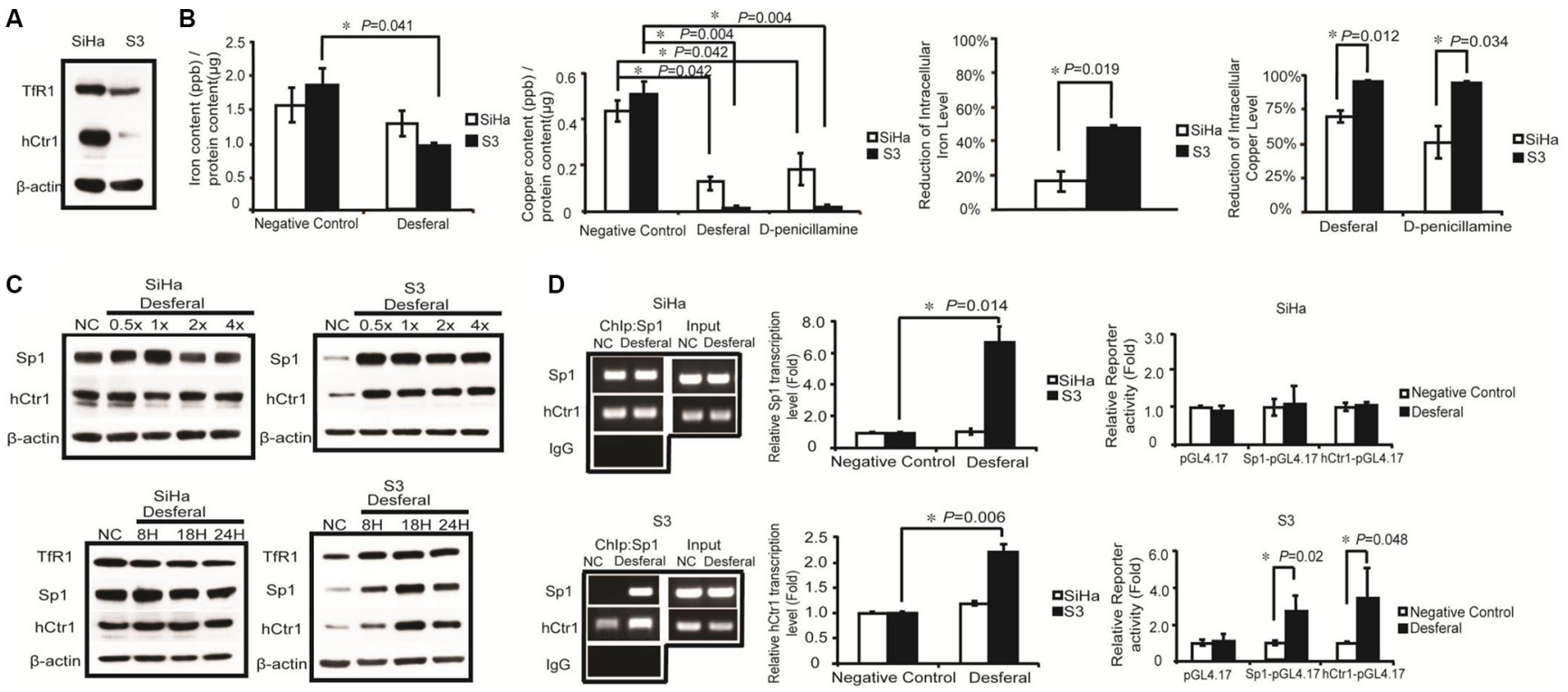

Figure 2: Effects of desferal on the concentration of intracellular iron and copper and expression of Sp1, hCtr1, and TfR1. (A) Western blot analysis of the expression levels of hCtr1 and TfR1 protein in SiHa and S3 cells. $\beta$-Actin served as an internal control. (B) SiHa and S3 cells were treated with 1-fold of $\mathrm{IC}_{50}$ of desferal or D-penicillamine. After $24 \mathrm{~h}$, cells were lysed and subjected to mass spectrometry for determining intracellular iron and copper concentrations $(P<0.05)$. (C) SiHa and S3 cells were treated with folds of $\mathrm{IC}_{50}$ of desferal for $24 \mathrm{~h}$. Cell lysates were then harvested and subjected to Western blot analysis. NC denotes negative control. SiHa and S3 cells were treated with 1-fold of $\mathrm{IC}_{50}$ of desferal for 8, 18, and $24 \mathrm{~h}$. Cell lysates were then harvested and subjected to Western blot analysis. NC denotes negative control. (D) The effect of desferal on Sp1 binding to the Sp1 and hCtr1 promoter regions in both SiHa and $\mathrm{S} 3$ cells was analyzed through ChIP. The effect of desferal on the transcription levels of both Sp1 and hCtr1 genes was determined through RT-qPCR $(P<0.05)$. The effect of desferal on the promoter activity levels of both Sp1 and hCtr1 promoters was determined using the promoter assay $(P<0.05)$. 
Consistent with these findings, we observed that desferal reduced intracellular copper concentrations and increased Sp1, hCtr1, and TfR1 expression levels both in a dose- and time-dependent manner in $\mathrm{S} 3$ cells (Figure 2C). Moreover, the ChIP assay revealed that Sp1 increased the binding of Sp1 to hCtr1 promoter regions and then increased Spl and hCtr1 transcription and translation in S3 cells treated with desferal (Figure 2D). Cumulative evidence has indicated that $\mathrm{Sp} 1$ directly regulates $\mathrm{NF}-\kappa \mathrm{B}$ p65 transcription $[15,16]$. By using QIAGEN EpiTectChIP promoter binding site prediction, we observed two putative NF- $\mathrm{B}$ p 65 binding sites on the TfR1 promoter. We then hypothesized that desferal affects TfR 1 expression by increasing the binding of Sp1

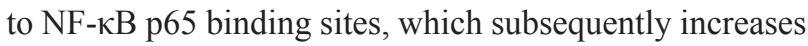
$\mathrm{NF}-\kappa \mathrm{B}$ p65 expression and binding to the TfR 1 promoter region. Our results revealed that desferal increased $N F-\kappa B$ p65 expression in S3 cells (Figure 3A). In addition, desferal treatment increased the nuclear accumulation of Sp1 and NF- $\kappa$ B p65 only in S3 cells (Figure 3B). Taken together, these data confirmed for the first time that an iron chelator could increase TfR 1 expression through the Sp1-NF- $\kappa$ B-TfR1 axis (Figure 3C and 3D). Compared the negative control from nuclear extraction assay (Figure 3B) and ChIP assay (Figure 3C), $\mathrm{Sp} 1$ in $\mathrm{S} 3$ cells presented the lower expression than in SiHa cells. Therefore, lower transcription of $\mathrm{Sp} 1$ in $\mathrm{S} 3$ cells caused the low expression of TfR1. Given the previous finding that transferrin receptor promoter contains several Spl-like binding sites [30], in this study we revealed that TfR1 expression was through the Sp1-NF-kB-TfR1 axis. Therefore, Sp1 may also directly bind transferrin receptor promoter and collaborate with NF-kB in upregulating transferrin receptor expression.

We assessed the antitumor activity of oxaliplatin in combination with or without desferal in human tumor xenografts in mice. Compared with $\mathrm{SiHa}$ tumors, the combination of oxaliplatin and desferal significantly increased the antitumor activity of oxaliplatin in $\mathrm{S} 3$ tumors (Figure 5A-5B). Furthermore, IHC analysis revealed that
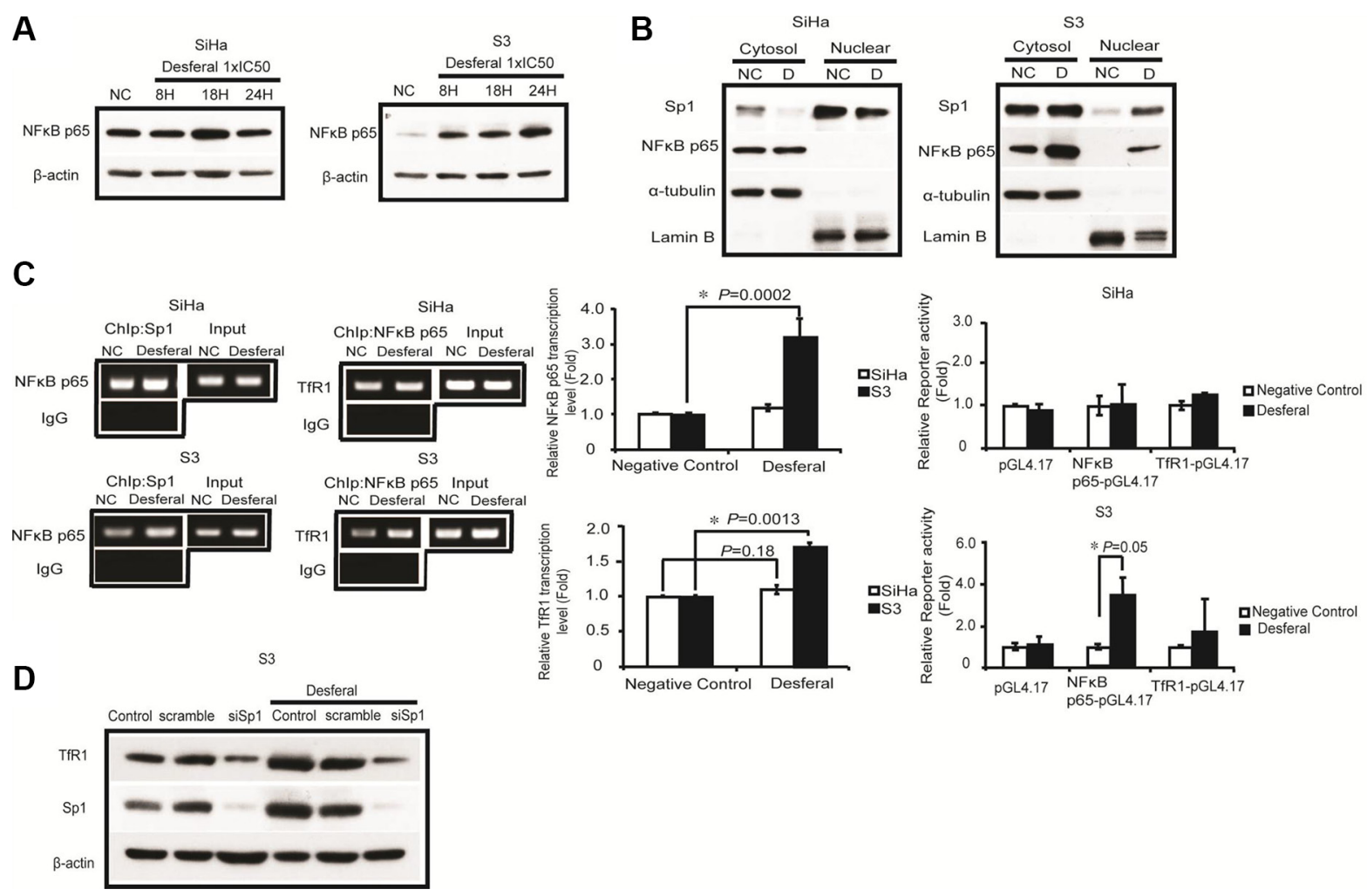

Figure 3: Desferal induced the expression level of TfR1 through the Sp1 -dependent pathway. (A) Desferal induced NF- $\mathrm{B}$ p65 expression in S3 cells in a time-dependent manner. Both SiHa and S3 cells were treated with desferal for 8, 18, and $24 \mathrm{~h}$. Cells were then harvested and subjected to Western blot analysis. NC denotes negative control (B) The effect of desferal on the nuclear localization of $\mathrm{Sp} 1$ and NF- $\mathrm{kB}$ p65. Cells were treated with or without desferal for $24 \mathrm{~h}$, and the cytosolic and nuclear fractions were then separated, as described in the Materials and Methods section. (C) Desferal induces the binding of Sp1 to the NF- $\mathrm{kB}$ p65 promoter region and that of NF$\kappa \mathrm{B}$ p65 to the TfR1 promoter region in $\mathrm{S} 3$ cells as demonstrated using a ChIP assay. Cells without desferal treatment served as the negative control (NC). Desferal significantly induced the transcription of NF- $\mathrm{B}$ p65 and TfR1 in S3 cells as demonstrated using an RT-qPCR assay. The effect of desferal on the promoter activity levels of both NF- $\kappa$ B p65 and TfR 1 promoters was determined using promoter assay $(P<0.05)$. (D) Desferal induced TfR1 expression through the Sp1-dependent pathway. S3 cells were transfected with either scramble control or Spl-targeted siRNA, respectively, followed by treatment with desferal. Cells without transfection served as the control. $\beta$-actin was used as an internal control. 
the expression levels of hCtr1 and TfR 1 increased only in S3 tumor tissues treated with desferal (Figure 5C-5D). These results suggest that desferal restores the sensitivity of cancer cells to platinum-based drugs through the upregulation of $\mathrm{hCtr} 1$ and $\mathrm{TfR} 1$. The transferrin receptor is responsible for major iron homeostasis and supports growth of cancer cells [31, 32]. Because malignant cells have increased transferrin receptor expression, this receptor is widely considered as an accessible portal for drug delivery into cancer cells and is becoming a potential target for cancer therapy [33-35]. A phase 1 clinical study reported a partial response of $87 \%$ after using cisplatinconjugated transferrin to treat patients with advanced cancers [36]. In addition, Fu et al. reported that in patients with ovarian cancer refractory to platinum drug treatment, platinum resistance was overcome through the use of a copper-lowering agent [37]. In addition to a copper chelator, our data provide evidence that an iron chelator can overcome platinum resistance through the regulation of hCtr1 and TfR1.

In conclusion, we demonstrated that the synergistic killing effect of the combination of desferal and oxaliplatin

A
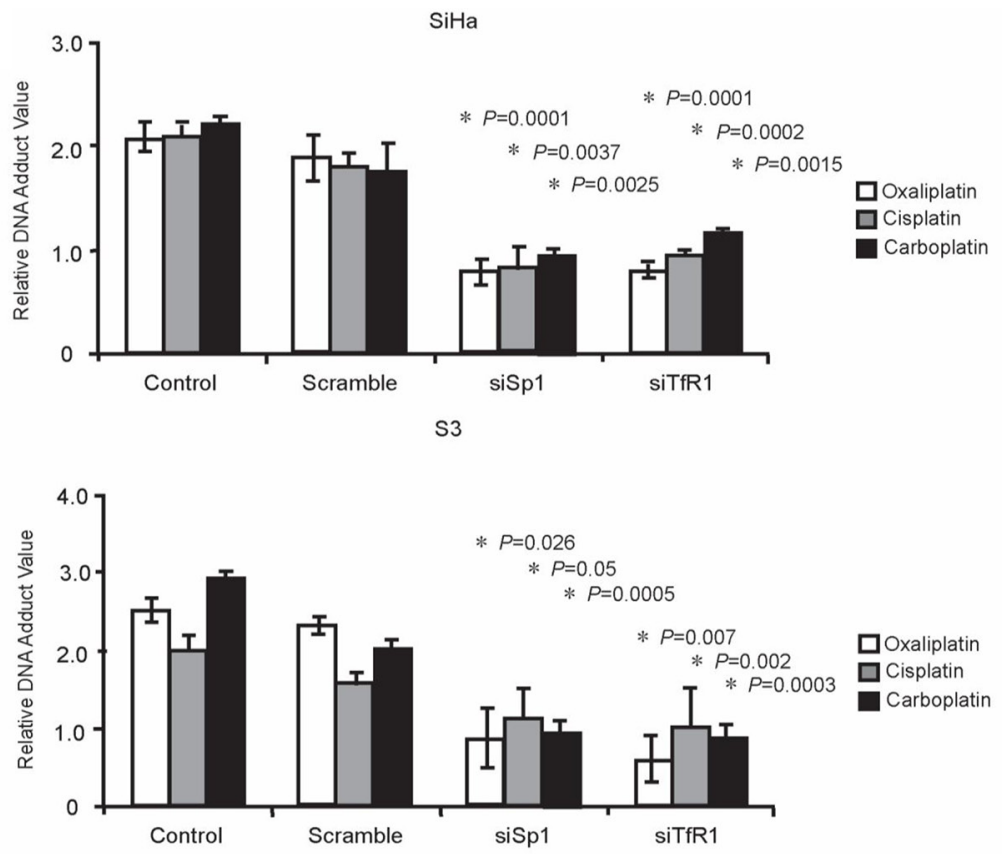

B $\mathrm{SiHa}$
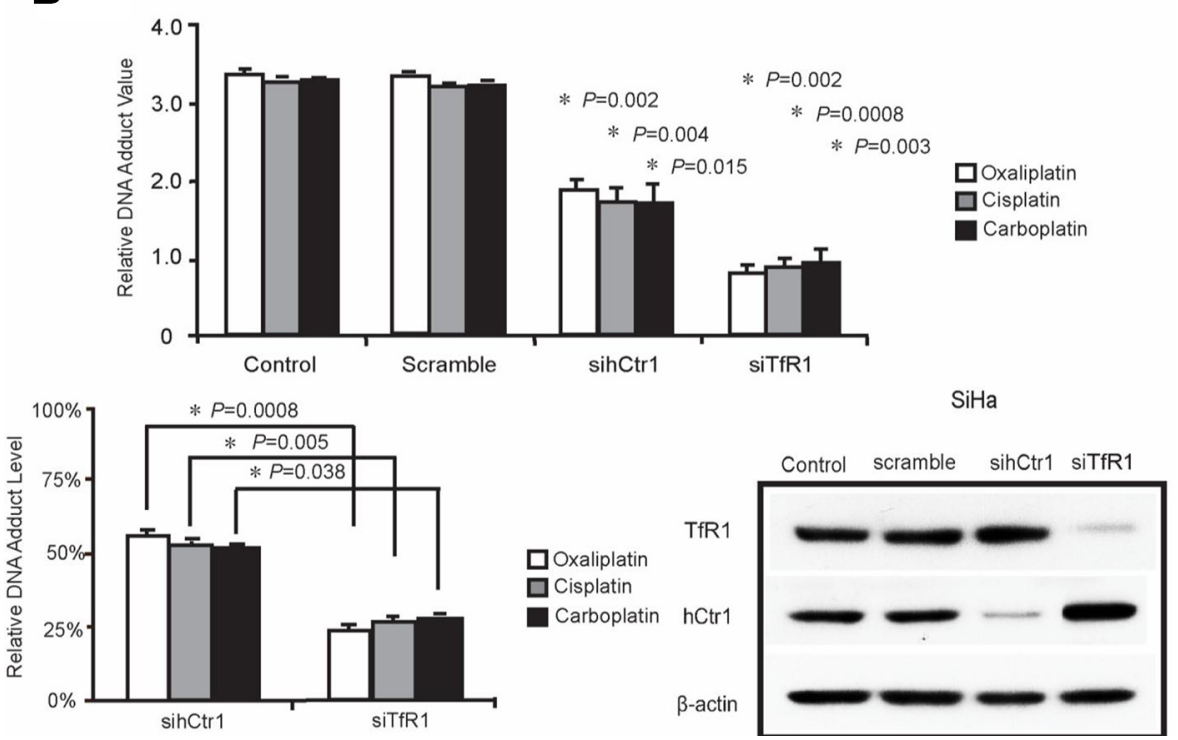

Figure 4: TfR1 promoted DNA adduct formation through Sp1. (A) Sp1 or TfR1-targeted were transfected into SiHa and S3 cells, respectively. 5-fold of $\mathrm{IC}_{50}$ platinum drugs including oxaliplatin, cisplatin, and carboplatin were incubated with cells for performing ELISA assay $(P<0.05)$, as described in Materials and Methods section. (B) SiHa cells were transfected with TfR1- or hCtr1-targeted siRNA for comparing the efficiency of transporting platinum drugs for performing ELISA assay $(P<0.05)$. 
A
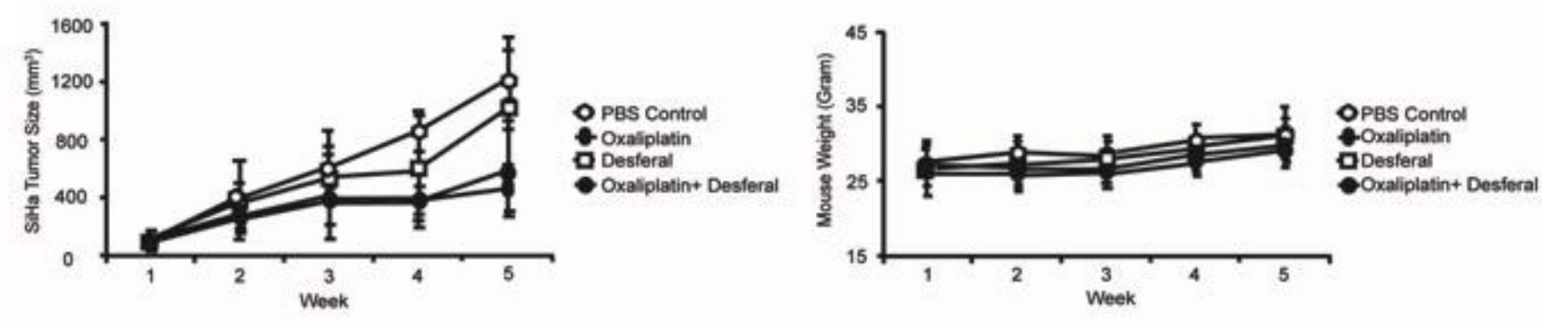

B
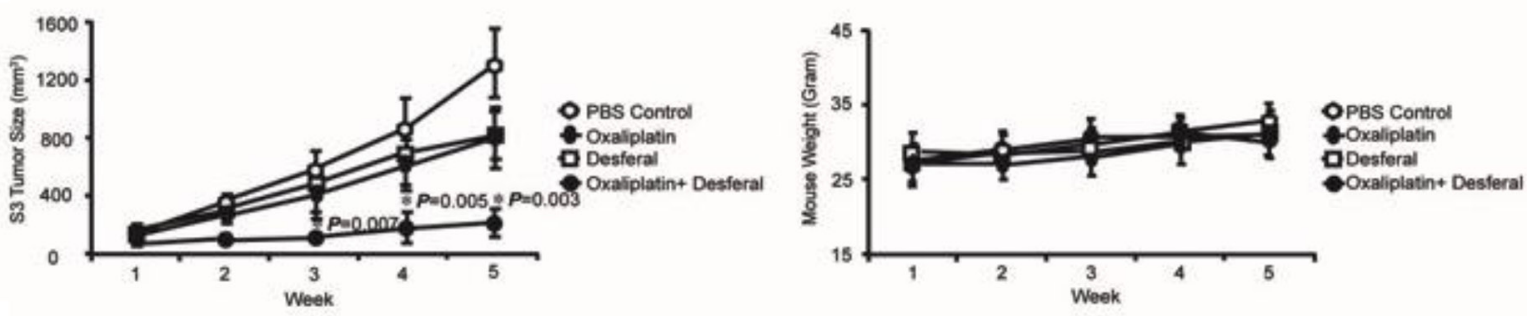

C

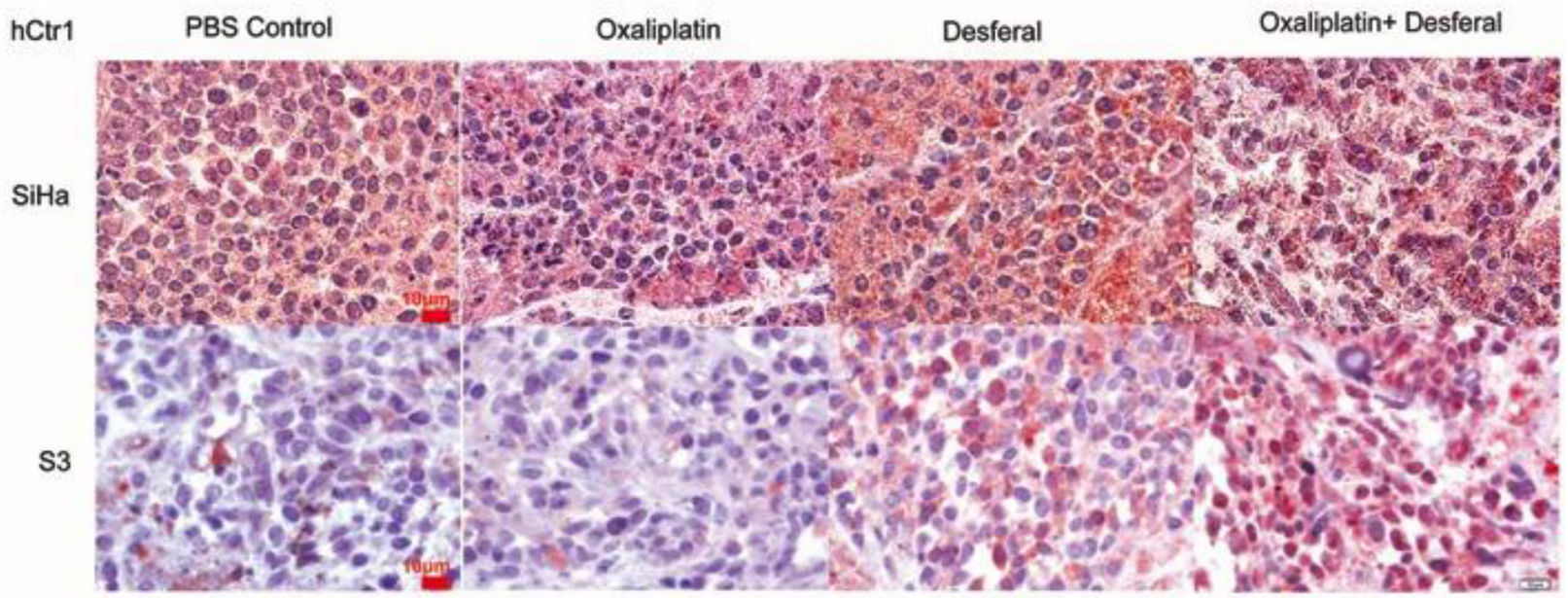

D

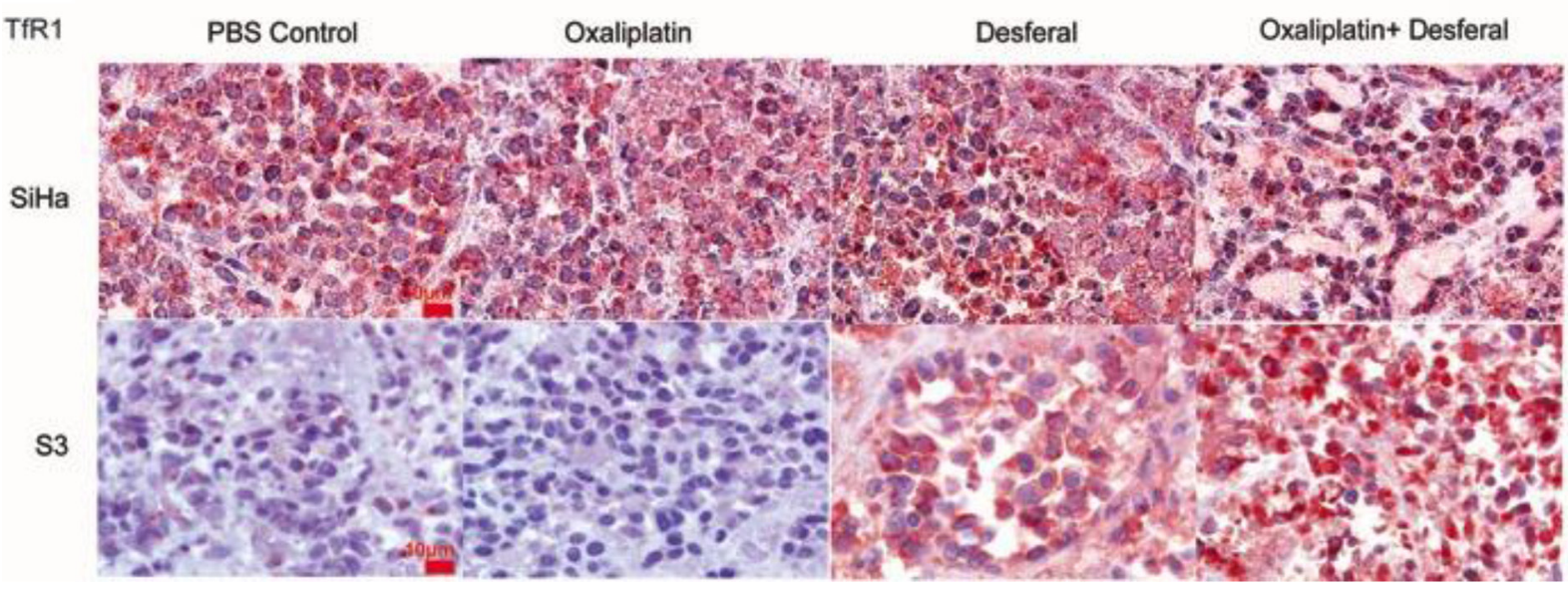

Figure 5 : The antitumoral effect of oxaliplatin alone or in combination with desferal in the xenograft animal model. Nude mice bearing SiHa cells (A) and S3 (B) cells were treated with PBS control $(\mathbf{O}), 5 \mathrm{mg} / \mathrm{kg}$ of oxaliplatin (-6), $200 \mathrm{mg} / \mathrm{kg}$ of desferal $(\square)$, or a combination of oxaliplatin and desferal $(-) 3$ times per week for 3 wk through a tail vein injection. Data are presented as the mean $\pm \mathrm{SD}$ of tumor volume $\left(\mathrm{mm}^{3}\right)$ of each time point $\left(n=5,{ }^{*} P<0.05\right)$. Immunohistochemistry analysis of hCtr1 (C) and TfR1 (D) expression in tumor tissues (1000× magnification). 
on oxaliplatin-resistant cells is through the modulation of the expression of a copper transporter and transferrin receptor in vitro and in vivo. In addition, we reported that the upregulation of transferrin receptor is through the Sp1-NF-кB-dependent pathway. These findings provide a potential therapeutic strategy for patients having oxaliplatin resistance and decreased hCtrl and TfR1 expression.

\section{MATERIALS AND METHODS}

\section{Reagents}

Platinum-based compounds, oxaliplatin, cisplatin, and carboplatin, were obtained from Sanofi (NY, USA, 4F121A), Fresenius Kabi (India, 871QZ01001), and Pharmachemie B.V. (Haarlem, Netherlands, 12D7MD), respectively. D-penicillamine (P4875-1G) and desferal (D9533-1G) were purchased from Sigma-Aldrich. Primary antibodies, hCtr1 (sc-66847) and NF- $\mathrm{BB}$ p65 (sc-109), were purchased from Santa Cruz (CA, USA); Sp1 (07-645), $\alpha$-tubulin (2508783), and TfR1 (ab84036) were purchased from Millipore Corporation (MA, USA) and Abcam (MA, USA), respectively. Horseradish peroxidase (HRP)conjugated secondary antibodies (sc-2055, sc-2054) were purchased from Santa Cruz (CA, USA).

\section{Cell lines and culture}

SiHa parental cells were from ATCC and cultured in DMEM medium (Thermo Scientific, Utah, USA, SH30003.02) supplemented with 5\% fetal bovine serum (Thermo Scientific, Utah, USA, SH30071.03), 100U/mL of penicillin, and $100 \mu \mathrm{g} / \mathrm{mL}$ of streptomycin-glutamate (Thermo Scientific, Utah, USA, 10378016) in a $5 \% \mathrm{CO}_{2}$ incubator (humidified atmosphere) at $37^{\circ} \mathrm{C}$. S3 cells, an oxaliplatin-resistant subclone of $\mathrm{SiHa}$ cells, were cultured in DMEM medium supplemented with $10 \%$ fetal bovine serum, $100 \mathrm{U} / \mathrm{mL}$ of penicillin, $100 \mu \mathrm{g} / \mathrm{mL}$ of streptomycin-glutamate, and $2 \mu \mathrm{g} / \mathrm{mL}$ of oxaliplatin. For the following assays, resistant cells were cultured in the medium without oxaliplatin to prevent the interference caused by oxaliplatin.

\section{Cell growth inhibition assay and combination index}

For the cell growth inhibition assay, both $\mathrm{SiHa}$ and S3 cell lines were subjected to desferal, oxaliplatin, cisplatin, or carboplatin treatment, respectively, for $72 \mathrm{~h}$. At the end of the treatment, cell growth was measured using a crystal violet stain assay. Cell growth inhibition determined by the dose that caused $50 \%$ cell death was designated as $\mathrm{IC}_{50}$. Resistance index was calculated using the following equation: $\left(\mathrm{IC}_{50}\right.$ of $\mathrm{S3}$ cells $) /\left(\mathrm{IC}_{50}\right.$ of $\mathrm{SiHa}$ cells). The CI between desferal and platinum drugs, including oxaliplatin, cisplatin, and carboplatin, was obtained using the Calcusyn software program, Version 1 (Biosoft, Cambridge, UK), which is based on the median effect equation of Chou and Talalay $[38,39]$. The analysis used in this study was under the assumption of mutual no exclusiveness of the mechanism of drug action. On the basis of the theory of Chou and Talalay, CI defines additive effect as $\mathrm{CI}=1$, synergism as $\mathrm{CI}<1$, and antagonism as $\mathrm{CI}>1$ in a combination of two drugs.

\section{DNA adduct measurement}

$\mathrm{SiHa}$ and $\mathrm{S} 3$ cells were treated with a 1 -fold $\mathrm{IC}_{50}$ dose of oxaliplatin, carboplatin, and cisplatin either alone or in combination with desferal for $6 \mathrm{~h}$. DNA was extracted and processed according to manufacturer instructions (Promega, Madison, USA, A1125). DNA adducts were measured using ELISA; each sample was coated on a 96-well ELISA plate (Sigma-Aldrich, CLS2525-10EA) and probed with specific monoclonal ICR4 antibodies (Millipore, MA, USA, Q2296671) to intrastrand adducts. For detection, 1-Step Ultra TMBELISA (Thermo Scientific, Utah, USA, PE1814394) was added to each sample and the reaction was stopped by adding $1 \mathrm{M}$ sulfuric acid. The absorption wavelength was measured at $450 \mathrm{~nm}$ by using a microplate reader (Spectramax M5 plate reader, Molecular Devices) [40].

\section{Measurement of intracellular iron and copper concentrations}

$\mathrm{SiHa}$ and $\mathrm{S} 3$ cell lysates were harvested using a lysis buffer (0.5\% NP-40 and 10\% glycerol in phosphatebuffered saline) before desferal or D-penicillamine treatment for $24 \mathrm{~h}$ or without treatment as a control. The protein concentration of whole-cell lysates was quantified and iron and copper concentrations were measured using Perkin Elmer SCIEX ELAN 6100 ICP mass spectrometer (Shelton, CT, USA).

\section{Small interference RNA transfection}

Sp1-, NF-кB p65-, and TfR1-specific human small interfering RNAs (siRNAs) were purchased from Invitrogen (Waltham, USA, s13318, s11914, and s727). siRNA oligonucleotides were introduced into cells by using the oligofectamine transfection reagent according to manufacturer instructions.

\section{Western blot analysis}

Cells were initially seeded at a density of $1 \times 10^{6}$ in $100-\mathrm{mm}^{2}$ dishes. After treatment, cells were lysed using a cell lysis buffer (Sigma-Aldrich, C2978) with 1 mM DTT, $1 \mathrm{mM}$ PMSF, and a protease inhibitor (Roche, Mannheim, Deutschland, 04693116001) and vortexed for 3 seconds 
then incubated on ice for $1 \mathrm{~h}$. Lysates were centrifuged at $4^{\circ} \mathrm{C}$ at $13,200 \mathrm{rpm}$ for 15 minutes. After centrifuging, supernatant was transferred to the new eppendorf. Further, $100 \mu \mathrm{g}$ of cell lysates were separated using $10 \%$ SDS-PAGE at $100 \mathrm{v}$ and were then transferred on polyvinylidinedifluoride membranes. The target proteins were probed with specific primary antibodies and HRPconjugated secondary antibodies. The immunoreactive bands were detected using the ECL method and visualized on Kodak Bio-MAX MR film.

\section{Chromatin immunoprecipitation PCR}

SiHa and S3 cells were treated with or without desferal for $24 \mathrm{~h}$ and then were crosslinked with $37 \%$ formaldehyde for $10 \mathrm{~min}$ at $37^{\circ} \mathrm{C}$. The cells were then harvested and processed for DNA fragmentation through sonication on ice. The resultant chromatin-DNA fractions were purified using a ChIP assay kit (Millipore, 2487694) according to manufacturer instructions. Chromatin was immunoprecipitated at $4^{\circ} \mathrm{C}$ by using anti-Sp1, anti$\mathrm{NF}-\kappa \mathrm{B}$ p65, and normal IgG (negative control). Primer sequences used were as follows: $\mathrm{Sp} 1$ on $\mathrm{Sp} 1$ promoter: forward 5'-CTTGGAGAGCAAGCGAGTCT-3', reverse 5'-TGGACTCATCCTTACCGCTC-3'; Sp1 on hCtr1 promoter: forward 5'-CGGTCTCTGGACCGA AAGTA-3', reverse 5'-CTCCGAATCTTAACCCGTCA-3'; Sp1 on NF- $\kappa$ B p65 promoter: forward 5'-AACTAATA ATAGGCCGGGCG-3', reverse 5'-CGTGTTAGCCAGG ATGGTTT-3'; and NF-кB p65 on TfR1 promoter: forward 5'-CTGCTTCTTGAGGGAAAACG-3', reverse 5'-TTAGG GGAGAACGCATCTGA-3'.

\section{Quantitative reverse transcription PCR}

Total RNA was extracted from SiHa and S3 cell lines by using TRIzol ${ }^{\circledR}$ reagent (Thermo Fisher, 74108). First-strand cDNA was synthesized using the Super Script III first strand synthesis system according to manufacturer instructions. The transcription level of Sp1, hCtr1, NF$\kappa \mathrm{B}$ p65, and TfR1 was quantified using the Applied Biosystems 7500 Real-Time PCR system, with RPL13A as an internal control. Primer sequences used were as follows: hCtr1: forward 5'-AAGGGTGGTGGAATAATTGCAG-3', reverse 5'-CTCACGAACTTGTGCCAGTAG-3'; Sp1: forward 5'-AGTTCCAGACCGTTGATGGG-3', reverse 5'-GTTTGCACCTGGTATGATCTGT-3'; NFкB p65: forward 5'- GCAGAGGGGAATGCGTTTTAG-3', reverse 5'-AGAAGGGTATGTTCGGTTGTTG-3'; and TfR1: forward 5'-ACCATTGTCATATACCCGGTTCA-3', reverse 5'-CAATAGCCCAAGTAGCCAATCAT-3'.

\section{Gene reporter assay}

The reporter gene plasmids of Sp1-, hCtr1-, TfR1-, and NF-кB p65-pGL4.17 were purchased from
GENEWIZ (product numbers: B24656-1/V125740, B24656/V125742, B26543-1/M76364, and B26543-2/ M76368). In brief, plasmids were introduced into cells by using the oligofectamine transfection reagent for $24 \mathrm{~h}$ and then processed using the Dual-Luciferase 1000 Assay system (Promega, Madison, USA, E1910) according to manufacturer protocol.

\section{Nuclear extraction assay}

Samples were lysed using $500 \mu \mathrm{L}$ of buffer A (10 mM HEPES, $1.5 \mathrm{mM} \mathrm{MgCl}, 10 \mathrm{mM} \mathrm{KCl}, 0.5 \mathrm{mM}$ DTT, and $0.05 \%$ NP-40; $\mathrm{pH} 7.9)$. After centrifugation at $3,000 \mathrm{rpm}$ for $10 \mathrm{~min}$ at $4^{\circ} \mathrm{C}$, the supernatant was collected as a cytosolic fraction. The pellet was washed three times with a wash buffer (10 mM HEPES, $1.5 \mathrm{mM} \mathrm{MgCl}_{2}$, and $10 \mathrm{mM} \mathrm{KCl}$; $\mathrm{pH} 7.9)$ and then lysed using $450 \mu \mathrm{L}$ of buffer B (5 mM HEPES, $1.5 \mathrm{mM} \mathrm{MgCl}_{2}, 0.2 \mathrm{mM}$ EDTA, $0.5 \mathrm{mM}$ DTT, $26 \%$ glycerol, and $300 \mathrm{mM} \mathrm{NaCl}$; pH 7.9). The supernatant was collected after centrifugation at $13,200 \mathrm{rpm}$ for $20 \mathrm{~min}$ at $4^{\circ} \mathrm{C}$ as a nuclear fraction. $100 \mu \mathrm{g}$ of cytosolic and nuclear fractions were subjected to gel electrophoresis and analyzed through immunoblotting.

\section{Animal experiments and immunohistochemistry study}

All the animal experiments were conducted according to the protocols of the Institutional Animal Care and Use Committee (American Association for Laboratory Animal Science). The male athymic (NCR nu/nu) nude mice (aged 3-4 wk) were housed in a pathogen-free environment. Animals were subcutaneously inoculated on the dorsal lumbosacral region with $1 \times 10^{7} \mathrm{SiHa}$ cells or $2 \times 10^{7} \mathrm{~S} 3$ cells in a total volume of $100 \mu \mathrm{L}$ with a growth-factor-reduced basement membrane. When the tumor volume reached $70-100 \mathrm{~mm}^{3}$, the animals were randomly divided into four groups, with 5 animals in each group. The mice were treated with indicated chemicals and drugs through a tail vein injection three times a week for subsequent 3 weeks. During the treatment, tumor volume and weight were evaluated three times per week. The tumor masses were removed on the fifth week of the study and were subjected to IHC staining procedures.

\section{Statistical analysis}

Student's $t$-test was used to analyze the quantitative and statistical results. All data were carried out in triplicate and expressed as a mean and standard deviation (SD). A $P$ value of $<0.05$ was considered statistically significant.

\section{ACKNOWLEDGMENTS AND FUNDING}

This study was supported by following grants from the following agencies: Department of Health, Taiwan 
(CA105-SP-01), National Health Research Institutes (CA-105-PP-22), National Research Program for Biopharmaceuticals (MOST 105-2325-B-400-001), and Ministry of Education, Taiwan, R.O.C. (Aim for the Top University Project at National Cheng Kung University).

\section{CONFLICTS OF INTEREST}

All authors declare no conflicts of interest.

\section{REFERENCES}

1. Wohrer SS, Raderer M, Hejna M. Palliative chemotherapy for advanced gastric cancer. Ann Oncol. 2004; 15:1585-1595.

2. Takahashi T, Saikawa Y, Nakamura R, Kumagai K, Takeuchi H, Kubota T, Kitagawa Y. Dynamic alteration of gene expression induced by anticancer-agent exposure in gastric cancer cell lines. Oncol Rep. 2009; 21:451-459.

3. Lin Y, Ueda J, Kikuchi S, Totsuka Y, Wei WQ, Qiao YL, Inoue M. Comparative epidemiology of gastric cancer between Japan and China. World J Gastroenterol. 2011; 17:4421-4428.

4. Kelland LR. Preclinical perspectives on platinum resistance. Drugs. 2000; 59:1-8; discussion 37-38.

5. Hall MD, Okabe M, Shen DW, Liang XJ, Gottesman MM. The role of cellular accumulation in determining sensitivity to platinum-based chemotherapy. Annu Rev Pharmacol Toxicol. 2008; 48:495-535.

6. Basu A, Krishnamurthy S. Cellular responses to Cisplatin-induced DNA damage. J Nucleic Acids. 2010; 2010.

7. Kuo MT, Chen HH, Song IS, Savaraj N, Ishikawa T. The roles of copper transporters in cisplatin resistance. Cancer Metastasis Rev. 2007; 26:71-83.

8. Howell SB, Safaei R, Larson CA, Sailor MJ. Copper transporters and the cellular pharmacology of the platinumcontaining cancer drugs. Mol Pharmacol. 2010; 77:887-894.

9. Klomp LW, Lin SJ, Yuan DS, Klausner RD, Culotta VC, Gitlin JD. Identification and functional expression of HAH1, a novel human gene involved in copper homeostasis. J Biol Chem. 1997; 272:9221-9226.

10. Katano K, Safaei R, Samimi G, Holzer A, Rochdi M, Howell SB. The copper export pump ATP7B modulates the cellular pharmacology of carboplatin in ovarian carcinoma cells. Mol Pharmacol. 2003; 64:466-473.

11. Samimi G, Katano K, Holzer AK, Safaei R, Howell SB. Modulation of the cellular pharmacology of cisplatin and its analogs by the copper exporters ATP7A and ATP7B. Mol Pharmacol. 2004; 66:25-32.

12. Safaei R, Howell SB. Copper transporters regulate the cellular pharmacology and sensitivity to Pt drugs. Crit Rev Oncol Hematol. 2005; 53:13-23.

13. Chen CC, Chen LT, Tsou TC, Pan WY, Kuo CC, Liu JF, Yeh SC, Tsai FY, Hsieh HP, Chang JY. Combined modalities of resistance in an oxaliplatin-resistant human gastric cancer cell line with enhanced sensitivity to 5-fluorouracil. Br J Cancer. 2007; 97:334-344.

14. Chen SJ, Kuo CC, Pan HY, Tsou TC, Yeh SC, Chang JY. Mechanistic basis of a combination D-penicillamine and platinum drugs synergistically inhibits tumor growth in oxaliplatin-resistant human cervical cancer cells in vitro and in vivo. Biochem Pharmacol. 2015; 95:28-37.

15. Perkins ND, Agranoff AB, Pascal E, Nabel GJ. An interaction between the DNA-binding domains of RelA(p65) and Sp1 mediates human immunodeficiency virus gene activation. Mol Cell Biol. 1994; 14:6570-6583.

16. Hirano F, Tanaka H, Hirano Y, Hiramoto M, Handa H, Makino I, Scheidereit C. Functional interference of Sp1 and NF-kappaB through the same DNA binding site. Mol Cell Biol. 1998; 18:1266-1274.

17. Tacchini L, Gammella E, De Ponti C, Recalcati S, Cairo G. Role of HIF-1 and NF-kappaB transcription factors in the modulation of transferrin receptor by inflammatory and antiinflammatory signals. J Biol Chem. 2008; 283:20674-20686.

18. Cappellini MD. Exjade(R) (deferasirox, ICL670) in the treatment of chronic iron overload associated with blood transfusion. Ther Clin Risk Manag. 2007; 3:291-299.

19. Porter JB. Optimizing iron chelation strategies in betathalassaemia major. Blood Rev. 2009; :S3-7.

20. Whitnall M, Howard J, Ponka P, Richardson DR. A class of iron chelators with a wide spectrum of potent antitumor activity that overcomes resistance to chemotherapeutics. Proc Natl Acad Sci U S A. 2006; 103:14901-14906.

21. Lovejoy DB, Jansson PJ, Brunk UT, Wong J, Ponka P, Richardson DR. Antitumor activity of metal-chelating compound $\mathrm{Dp} 44 \mathrm{mT}$ is mediated by formation of a redoxactive copper complex that accumulates in lysosomes. Cancer Res. 2011; 71:5871-5880.

22. Lui GY, Obeidy P, Ford SJ, Tselepis C, Sharp DM, Jansson PJ, Kalinowski DS, Kovacevic Z, Lovejoy DB, Richardson DR. The iron chelator, deferasirox, as a novel strategy for cancer treatment: oral activity against human lung tumor xenografts and molecular mechanism of action. Mol Pharmacol. 2013; 83:179-190.

23. Van Reyk DM, Dean RT. The iron-selective chelator desferal can reduce chelated copper. Free Radic Res. 1996; 24:55-60.

24. Safi R, Nelson ER, Chitneni SK, Franz KJ, George DJ, Zalutsky MR, McDonnell DP. Copper signaling axis as a target for prostate cancer therapeutics. Cancer Res. 2014; 74:5819-5831.

25. Chen HH, Chen WC, Liang ZD, Tsai WB, Long Y, Aiba I, Fu S, Broaddus R, Liu J, Feun LG, Savaraj N, Kuo MT. Targeting drug transport mechanisms for improving platinum-based cancer chemotherapy. Expert Opin Ther Targets. 2015; 19:1307-1317.

26. Kilari D, Guancial E, Kim ES. Role of copper transporters in platinum resistance. World J Clin Oncol. 2016; 7:106-113. 
27. Ishida S, Lee J, Thiele DJ, Herskowitz I. Uptake of the anticancer drug cisplatin mediated by the copper transporter Ctr1 in yeast and mammals. Proc Natl Acad Sci U S A. 2002; 99:14298-14302.

28. Lee J, Prohaska JR, Dagenais SL, Glover TW, Thiele DJ. Isolation of a murine copper transporter gene, tissue specific expression and functional complementation of a yeast copper transport mutant. Gene. 2000; 254:87-96.

29. Helleman J, Burger H, Hamelers IH, Boersma AW, de Kroon AI, Stoter G, Nooter K. Impaired cisplatin influx in an A2780 mutant cell line: evidence for a putative, cisconfiguration-specific, platinum influx transporter. Cancer Biol Ther. 2006; 5:943-949.

30. Casey JL, Di Jeso B, Rao K, Rouault TA, Klausner RD, Harford JB. The promoter region of the human transferrin receptor gene. Ann N Y Acad Sci. 1988; 526:54-64.

31. Hann HW, Stahlhut MW, Blumberg BS. Iron nutrition and tumor growth: decreased tumor growth in iron-deficient mice. Cancer Res. 1988; 48:4168-4170.

32. Torti SV,Torti FM. Iron and cancer: more ore to be mined. Nat Rev Cancer. 2013; 13:342-355.

33. Prost AC, Menegaux F, Langlois P, Vidal JM, Koulibaly M, Jost JL, Duron JJ, Chigot JP, Vayre P, Aurengo A, Legrand JC, Rosselin G, Gespach C. Differential transferrin receptor density in human colorectal cancer: A potential probe for diagnosis and therapy. Int J Oncol. 1998; 13:871-875.
34. Shinohara H, Fan D, Ozawa S, Yano S, Van Arsdell M, Viner JL, Beers R, Pastan I, Fidler IJ. Site-specific expression of transferrin receptor by human colon cancer cells directly correlates with eradication by antitransferrin recombinant immunotoxin. Int J Oncol. 2000; 17:643-651.

35. Gomme PT, McCann KB, Bertolini J. Transferrin: structure, function and potential therapeutic actions. Drug Discov Today. 2005; 10:267-273.

36. Head JF, Wang F, Elliott RL. Antineoplastic drugs that interfere with iron metabolism in cancer cells. Adv Enzyme Regul. 1997; 37:147-169.

37. Fu S, Naing A, Fu C, Kuo MT, Kurzrock R. Overcoming platinum resistance through the use of a copper-lowering agent. Mol Cancer Ther. 2012; 11:1221-1225.

38. Chou TC. Theoretical basis, experimental design, and computerized simulation of synergism and antagonism in drug combination studies. Pharmacol Rev. 2006; 58:621-681.

39. Chou TC. Drug combination studies and their synergy quantification using the Chou-Talalay method. Cancer Res. 2010; 70:440-446.

40. Chen SH, Kuo CC, Li CF, Cheung CH, Tsou TC, Chiang HC, Yang YN, Chang SL, Lin LC, Pan HY, Chang KY, Chang JY. $\mathrm{O}^{6}$-methylguanine DNA methyltransferase repairs platinum-DNA adducts following cisplatin treatment and predicts prognoses of nasopharyngeal carcinoma. Int $\mathrm{J}$ Cancer. 2015; 137:1291-1305. 